\title{
Abordaje anterolateral para artroplastia total de cadera
}

\section{Anterolateral approach to the hip arthroplasty}

\author{
Humberto González Ugalde, ${ }^{*}$ Héctor Antonio Soriano Solís ${ }^{\ddagger}$
}

Citar como: González UH, Soriano SHA. Abordaje anterolateral para artroplastia total de cadera. An Med ABC. $2021 ; 66$ (2): $110-113$. https://dx.doi.org/10.35366/100479

\begin{abstract}
RESUMEN
Los abordajes para la artroplastia de cadera siempre generan controversia, ya que los resultados de los estudios son similares en cuanto a tasas de éxito y complicaciones. El abordaje lateral es uno de los más utilizados en el mundo con excelentes resultados funcionales y bajos índices de complicación. Se expondrán los antecedentes del abordaje, las ventajas y complicaciones principales, así como las preferencias del autor.
\end{abstract}

Palabras clave: Abordajes, artroplastia, anterolateral.

Nivel de evidencia: III

\section{INTRODUCCIÓN}

Históricamente el abordaje anterolateral está basado en los principios anatómicos y quirúrgicos del abordaje lateral directo para el tratamiento de las fracturas del cuello femoral, el cual fue descrito por Watson-Jones en Inglaterra en 1936, en el que se realizaba una desinserción total o parcial de las

* Cirujano Ortopedista. Cirugía Articular y Reconstrucción Articular de Cadera y Rodilla.

‡ Cirujano Ortopedista. Reconstrucción Articular de Cadera y Rodilla.

Centro Médico ABC Santa Fe. Ciudad de México.

Recibido: 02/05/2021. Aceptado: 08/06/2021.

Correspondencia:

Dr. Humberto González Ugalde

E-mail: humbertogonzalezmd@gmail.com

\section{ABSTRACT}

Approaches to hip replacement are always controversial as studies results are similar, in terms of success and complication rates. The lateral approach is one of the most used in the world with excellent functional results and low complication rates. The background of the approach, the main advantages and complications, as well as the author's preferences will be explained.

Keywords: Approach, arthroplasty, anterolateral.

Level of evidence: III

fibras del glúteo medio y menor, y una osteotomía trocantérica con la finalidad de visualizar el cuello femoral. ${ }^{1}$

En 1982 Kevin Hardinge populariza el abordaje lateral directo, antes descrito en 1954 por McFarland y Osborne para la colocación de prótesis de cadera. Más adelante se realizaron distintas modificaciones a los abordajes con la finalidad de hacerlo menos invasivo e igual de efectivo. Y así, con este mismo sentido, el abordaje lateral ha evolucionado y se ha convertido en una técnica con menor invasión, menos daño a los tejidos, la misma visualización y reproducible, lo que lo hace un abordaje completo y versátil. ${ }^{2}$

\section{TÉCNICA QUIRÚRGICA}

Las referencias anatómicas para el abordaje lateral son la espina iliaca anterosuperior, el trocánter ma- 
yor y la diáfisis femoral. Con base en la evolución del abordaje, la incisión ha sido más pequeña, por lo que se realiza una incisión recta de aproximadamente 8 $\mathrm{cm}$ distal al trocánter mayor en el borde anterior de la diáfisis femoral y $4 \mathrm{~cm}$ proximal sobre la piel del paciente (dependiente de la complexión). Una vez realizada, se visualiza la bursa trocantérica, la cual se aparta para visualizar las fibras del vasto lateral y el glúteo medio, el vasto lateral se incide de manera recta y se levanta un colgajo muscular hacia adelante, la incisión se curva ligeramente sobre el trocánter y se continúa hacia proximal de manera roma dejando parte del tendón inserto sobre el trocánter mayor como referencia para cerrarlo, se diseca y separa hacia adelante exponiendo al glúteo menor, el cual se incide y se expone la cápsula, se corta de manera longitudinal, está descrito que no es necesaria la capsulectomía. ${ }^{3}$

Se han hecho modificaciones a este abordaje como en el grupo del Dr. Ilizaliturri y colaboradores, quienes realizan cambios a la técnicas en cuanto al tamaño de herida (llamado mínimo invasivo) que se reporta de $8.2 \pm 1.04 \mathrm{~cm}$ y en la disección del glúteo medio, el cual se desinserta siguiendo las fibras de éste y se refiere hacia anterior, se separa del glúteo menor, el cual posteriormente será desinsertado y referido anterior por separado, lo que nos aporta una mejor reconstrucción del aparato abductor, y con esto la disminución en la claudicación postoperatoria. ${ }^{4}$

En la Figura 1 se observan las referencias anatómicas para el abordaje y en la Figura 2 se observa cómo se identifican las estructuras anatómicas por planos hasta llegar a la cabeza femoral.

\section{Complicaciones}

Varios estudios han descrito este parámetro, pero enfatiza el del grupo de Melbourne, Australia, donde se describen claramente las complicaciones tempranas derivadas del abordaje en el que evaluaron 1,413 artroplastias de cadera tratadas por abordaje lateral y se midieron variables como luxación, infección, fracturas; en esa revisión no se encontró diferencia estadísticamente significativa comparado con los demás abordajes. $^{5}$

\section{RESULTADOS REPORTADOS}

Aunque sabemos que la satisfacción postoperatoria de la artroplastia de cadera oscila entre 89 y $95 \%$ de los pacientes, los abordajes toman importancia al ha- blar de complicaciones o de funciones sobre los pacientes como lo reporta Restrepo, quien realizó una evaluación SF-36, WOMAC y HHS a las seis semanas, seis meses y un año, donde claramente no hay diferencia más allá del primer año. 6,7

En los resultados funcionales reportados por Annik den Daas y colaboradores, en 48 caderas operadas por abordaje lateral, tomando en cuenta la escala de hip disability and osteoarthritis outcome scores, mejoraron considerablemente al igual que en el grupo control (abordaje anterior), por lo que no hubo diferencias estadísticamente significativas. ${ }^{8}$

En un estudio de la New York University Medical Center, realizado por Aggarwal y su equipo, en el que 3,574 pacientes fueron sometidos a una artroplastia total de cadera, reportaron sus estadísticas postoperatorias inmediatas, como tiempo promedio de cirugía, en el que el abordaje lateral oscila entre 68 y 92 minutos, así como la pérdida de sangre de 270 a $345 \mathrm{~cm}^{3}$, días de estancia hospitalaria entre 2.3 y 3.4 días, con un porcentaje de complicaciones entre 6.11 y $6.7 \%$. Y se reportaron complicaciones de infecciones superficiales de $1.9 \%$, infecciones profundas de $1.9 \%$, herida productiva de $2.3 \%$, fractura periprotésica de $2.7 \%$, luxación de $0 \%$, aflojamiento aséptico de $0.4 \%$ y el reingreso a quirófano por cualquier causa fue de $8.3 \% .^{9}$



Figura 1: Referencias para abordaje anterolateral.

EIAS = espina iliaca anterosuperior; TM = trocánter mayor; ASIS = espina iliaca anterior y superior. 

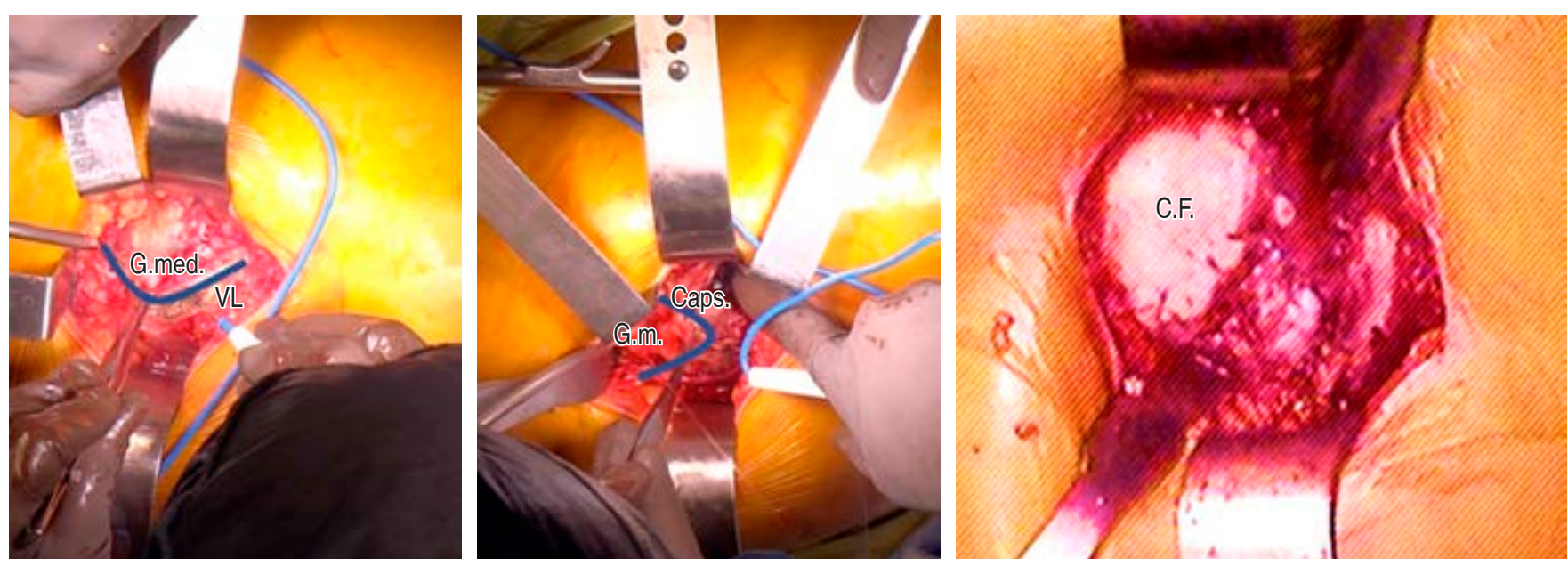

Figura 2: Secuencia de planos en el abordaje hasta llegar a la cabeza femoral.

G. med. = glúteo medio; VL = vasto lateral; $G$.m. = glúteo menor; $C F=$ cabeza femoral; Caps. = cápsula .

\section{DISCUSIÓN}

El abordaje lateral de cadera tiene una gran importancia por ser históricamente muy utilizado en patologías tanto degenerativas como traumáticas, además de tener buenos resultados funcionales y con las modificaciones del clásico abordaje se está buscando la mejora en tiempo quirúrgico, disminución del sangrado y complicaciones, sin olvidar la curva de aprendizaje.

La versatilidad, fácil comprensión de la anatomía y la reproducibilidad lo hace un excelente abordaje para el tratamiento de diversas patologías tanto degenerativas como traumáticas.

Un punto muy importante en cuanto a abordajes de cadera es que cada uno tiene sus ventajas específicas y se puede tomar una preferencia por el abordaje con el que más se sienta cómodo el cirujano, que logre cumplir su curva de aprendizaje y pueda perfeccionar los detalles para mejorar la recuperación del paciente.

Basándonos en la claudicación (signo clínico que se critica del abordaje anterolateral) y en el artículo del Dr. Masonis y colaboradores se concluye que no existe una gran diferencia en los resultados clínicos comparando el abordaje posterior, en el que se preserva el aparato abductor, y el abordaje anterolateral, el cual oscila entre $0-16 \%$ contra $4-20 \%$, lo cual no es clínica ni estadísticamente significativo. ${ }^{10}$ Asimismo, el grupo de Restrepo reporta que no hay diferencia en las escalas funcionales a largo plazo contra el abordaje anterior directo. ${ }^{7}$

En conclusión, los cirujanos ortopedistas que se dedican a la reconstrucción articular deben estar entrenados en los tres abordajes para resolver cualquier patología, ya sea primaria o de revisión.

\section{Preferencias del autor}

He tenido la oportunidad de experimentar con los tres abordajes para artroplastia de cadera y cada uno tiene sus ventajas, las cuales me hacen en ocasiones considerar cambiar a esa opción; pero los resultados funcionales, la capacidad de extensión del abordaje y la visualización que tengo con el abordaje anterolateral por mínima invasión, me hace seguir mi práctica con este abordaje.

\section{REFERENCIAS}

1. Jones RW, Roberts RE. Calcification, decalcification, and ossification*. Br J Surg [Internet]. 1934; 21 (83): 461-99. Available from: https://academic.oup.com/bjs/ article/21/83/461-499/6221212

2. Parker MJ. Fractures of the neck of the femur. Trauma [Internet]. 2008; 10 (1): 43-53. Available from: http://journals. sagepub.com/doi/10.1177/1460408608089640

3. Foster DE, Hunter JR. The direct lateral approach to the hip for arthroplasty. Advantages and complications. Orthopedics [Internet]. 1987; 10 (2): 274-280. Available from: http://journals.healio.com/doi/10.3928/0147-744719870201-06

4. Ilizaliturri VM Jr, Chaidez PA, Valero FS, Aguilera JM. Small incision total hip replacement by the lateral approach using standard instruments. Orthopedics. 2004; 27 (4): 377-381.

5. Hoskins W, Dowsey MM, Spelman T, Choong PFM. Early surgical complications of total hip arthroplasty related to surgical approach. ANZ J Surg. 2020; 90 (10): 2050-2055.

6. Specht K, Kjaersgaard-Andersen P, Kehlet H, Wedderkopp N, Pedersen BD. High patient satisfaction in 445 patients who 
underwent fast-track hip or knee replacement. Acta Orthop. 2015; 86 (6): 702-707.

7. Restrepo C, Parvizi J, Pour AE, Hozack WJ. Prospective randomized study of two surgical approaches for total hip arthroplasty. J Arthroplasty [Internet]. 2010; 25 (5): 671-9.e1. Available from: http://dx.doi.org/10.1016/j.arth.2010.02.002

8. den Daas A, Reitsma EA, Knobben BAS, Ten Have BLEF, Somford MP. Patient satisfaction in different approaches for total hip arthroplasty. Orthop Traumatol Surg Res
[Internet]. 2019; 105 (7): 1277-1282. Available from: https:// doi.org/10.1016/j.otsr.2019.08.003

9. Aggarwal VK, Elbuluk A, Dundon J, Herrero C, Hernandez C, Vigdorchik JM et al. Surgical approach significantly affects the complication rates associated with total hip arthroplasty. Bone Joint J. 2019; 101-B (6): 646-651.

10. Masonis JL, Bourne RB. Surgical approach, abductor function, and total hip arthroplasty dislocation. Clin Orthop Relat Res. 2002; (405): 46-53. 